\title{
Objects Are Highlighted by Spatial Attention
}

\author{
A. Martínez ${ }^{1,2}$, W. Teder-Sälejärvi ${ }^{1}$, M. Vazquez $^{3}$, S. Molholm ${ }^{2}$, \\ J. J. Foxe ${ }^{2,4}$, D. C. Javitt ${ }^{2,4}$, F. Di Russo ${ }^{5}$, M. S. Worden ${ }^{6}$, \\ and S. A. Hillyard ${ }^{1}$
}

\begin{abstract}
Selective attention may be focused upon a region of interest within the visual surroundings, thereby improving the perceptual quality of stimuli at that location. It has been debated whether this spatially selective mechanism plays a role in the attentive selection of whole objects in a visual scene. The relationship between spatial and object-selective attention was investigated here through recordings of event-related brain potentials (ERPs) supplemented with functional magnetic brain imaging (fMRI). Subjects viewed a display consisting of two bar-shaped objects and directed attention to sequences of stimuli (brief corner offsets) at one end of one of
\end{abstract}

\section{INTRODUCTION}

When attention is directed to a specific location in visual space, the detection and discrimination of stimuli within the focus of attention occurs faster and with greater accuracy than at unattended locations (Wright, 1998). Physiological studies have found that this spatial focusing of attention facilitates the processing of attendedlocation stimuli at an early sensory level (Maunsell \& McAdams, 2000; Hillyard \& Anllo-Vento, 1998). There is increasing evidence that visual attention may also select whole objects as integrated feature ensembles (Driver, Davis, Russell, Turatto, \& Freeman, 2001; Scholl, 2001). Support for object-based attention mechanisms has been derived from cued reaction time paradigms in which relevant and irrelevant stimuli belong to the same object or to different objects. In their now classic study, Egly, Driver, and Rafal (1994) presented subjects with a display of two horizontally or vertically oriented bars and cued one of the four ends as the most likely location where a subsequent target would appear. Reaction times (RTs) were found to be fastest to targets appearing at the attended (cued) location, but RTs were faster for targets presented at the uncued end of the attended bar than at a comparably distant uncued location on the

\footnotetext{
${ }^{1}$ University of California, ${ }^{2}$ Nathan S. Kline Institute for Psychiatric Research, ${ }^{3}$ Universidad de Sevilla, ${ }^{4}$ City College of the City University of New York, ${ }^{5}$ Fondazione Santa Lucia, ${ }^{6}$ Weill Medical College of Cornell University
}

the bars. Unattended stimuli belonging to the same object as the attended stimuli elicited spatiotemporal patterns of neural activity in the visual cortex closely resembling those elicited by the attended stimuli themselves, albeit smaller in amplitude. This enhanced neural activity associated with object-selective attention was localized by use of ERP dipole modeling and fMRI to the lateral occipital extrastriate cortex. We conclude that object-selective attention shares a common neural mechanism with spatial attention that entails the facilitation of sensory processing of stimuli within the boundaries of an attended object.

other bar. This "same-object advantage" has been replicated in numerous experiments patterned after that of Egly et al. (Lamy \& Egeth, 2002; Shomstein \& Yantis, 2002; Abrams \& Law, 2000; Avrahami, 1999).

Two major hypotheses have been put forth to account for the same-object advantage in cued RT experiments. According to one formulation, when spatial attention is directed to one part of an object the resulting top-down facilitation of early sensory processing spreads to encompass other regions within the object's boundaries (Davis, Driver, Pavani, \& Shepherd, 2000; Weber, Kramer, \& Miller, 1997; Vecera \& Farah, 1994). This "objectguided spatial selection" is proposed to strengthen the sensory representation of the entire object. A contrasting hypothesis accounts for the same-object advantage by proposing that locations within attended objects are given higher priority for target search (Yantis \& Serences, 2003; Shomstein \& Yantis, 2002). Thus, if a target is not found at the cued location, other locations within the cued object's boundaries are searched before locations on uncued objects. This "attentional prioritization" mechanism is proposed to control the order of locations to be visually investigated and not to have an influence on early sensory-level processing.

To decide between these alternative hypotheses, a key question is whether object- and space-based attention share a common physiological mechanism and, in particular, whether stimulus selection occurs at the same level of processing in the two cases. If spatial- 
and object-selective attention were found to modulate sensory processing at the same level of the visual pathways, this would support the object-guided spatial-selection hypothesis. The neural mechanisms of spatial- and object-selective attention have recently been compared using functional magnetic resonance imaging (fMRI) in an Egly-style paradigm (Müller \& Kleinschmidt, 2003). It was found that central cuing of a target's probable location at one end of a bar-shaped object produced an increase of neural activity in early visual cortical areas, not only at the retinotopic representation of the cued location but also (to a lesser extent) at the cortical representation of the other uncued end of the bar. Müller and Kleinschmidt (2003) proposed that this pattern of anticipatory activity reflected an object-based spatial selection interacting with an object-independent spatial selection mechanism in directing attention. On invalid trials, when the target did not appear at the cued location, there was greater activation in early cortical areas representing the uncued end of the cued object than in areas representing equidistant locations on another object. This effect was taken as evidence that objects guide spatial search for target information on invalid trials, consistent with object-based prioritized search models (e.g., Shomstein \& Yantis, 2002; Moore, Yantis, \& Vaughan, 1998).

The slow time course of the hemodynamic response imposes certain limitations on the conclusions that may be reached from fMRI studies of cued attention tasks of this type. In particular, it is difficult to distinguish neural activity associated with top-down attentional control or allocation processes from that associated with the facilitated processing of attended sensory inputs-the latter being the defining hallmark of a selective attention process. The poor time resolution of fMRI also makes it difficult to determine at which level of the sensory pathways the selection of attended input first occurs (Martinez et al., 1999). For example, if multiple cortical areas are modulated by attention, it is not clear whether selection occurs early and is fed forward to higher areas or whether it occurs at a later stage and is fed back. These questions may be investigated by use of measures of neural activity that have higher temporal resolution, such as event-related potentials (ERPs) or event-related magnetic fields (ERFs).

He, Fan, Shou, and Chen (2004) recorded ERPs to the targets in an Egly-style paradigm in which a peripheral cue directed attention to the probable target location at one end of a bar-shaped object. It was observed that an anteriorly distributed N1 component at 130-150 msec was preferentially enhanced in response to targets at the cued location, thereby indexing a space-based selection, whereas a posteriorly distributed N1 at 150-180 msec was enhanced for targets at both cued and uncued locations within the bar-shaped object, thus reflecting an object-based selection. The authors concluded that object selection occurs at an early sensory processing stage in the extrastriate visual pathways when a peripheral cue directs attention. The behavioral data obtained by $\mathrm{He}$ et al. further suggested that object-selective attention was allocated by a "reflexive mechanism" that operates under both high and low cue validity conditions, whereas spatial-selective attention was allocated by a "voluntary mechanism" that depended on high cue validity. Accordingly, the authors proposed that the voluntary mechanism engaged by high-validity spatial cueing does not result in object-based selection.

The present study used ERP recordings to demonstrate that voluntary, endogenously cued spatial attention can produce an object-based sensory facilitation under appropriate conditions. In our design, attention was directed by a central cue to continuous sequences of stimuli in one visual field quadrant, with horizontal or vertical bar-shaped objects linking the attended quadrant to stimulus sequences in another quadrant. In this way it could be determined whether the voluntary allocation of spatial attention to stimuli at one end of a bar would facilitate the processing (as indexed by ERP amplitude modulation) of stimuli at the opposite end of the same bar. To investigate whether spatial- and objectbased attention may share a common mechanism, which would support the object-based spatial selection hypothesis, we made a direct comparison between the spatiotemporal ERP patterns associated with each type of selection. The neural generators of ERP components reflecting object- and spatial-selective attention were then localized by use of dipole modeling and compared with mappings of attention-related activity obtained by fMRI to identify the participating visual cortical regions. Finally, because this paradigm involved sustained attention to one location rather than a trial-by-trial cuing with valid and invalid trials, it was possible to rule out a mechanism of prioritized target search to account for the object-selective ERP modulations that were observed.

\section{RESULTS}

Subjects viewed a display consisting of two rectangular bars oriented either vertically or horizontally (Figure 1). The task stimuli consisted of brief offsets of the corners of the bars, which occurred one at a time in a rapid, randomized sequence. During each 20 -sec run the corner offsets in one of the four quadrants were designated as relevant and had to be discriminated for shape. Target discrimination accuracy averaged $92.4 \%$ correct detections (with an average false alarm rate of 16.0\%) and did not differ significantly among the quadrants. Spatial attention effects were assessed by comparing brain activity (both ERP and blood oxygenation level dependent [BOLD] responses) in each quadrant elicited by stimuli when they were attended to the activity elicited by the same stimuli when unattended. Object-selective effects were assessed for each quadrant by comparing 
Figure 1. Experimental design. During each block, two horizontal or vertical bars were continuously present on the screen. Subjects maintained fixation on a central cross and covertly attended to the visual quadrant indicated by a pair of arrows directly above or below fixation. Stimuli were brief (100 msec) offsets of the corners of the bars leaving either a concave (standard) or convex (target) edge (see inlay). Analysis of objectselective attention effects focused on the response elicited by unattended corner offsets as a function of whether these formed part of the attended bar (same object condition, vertical bars in the example shown) or belonged to the unattended bar (different object condition, horizontal bars in the example shown).

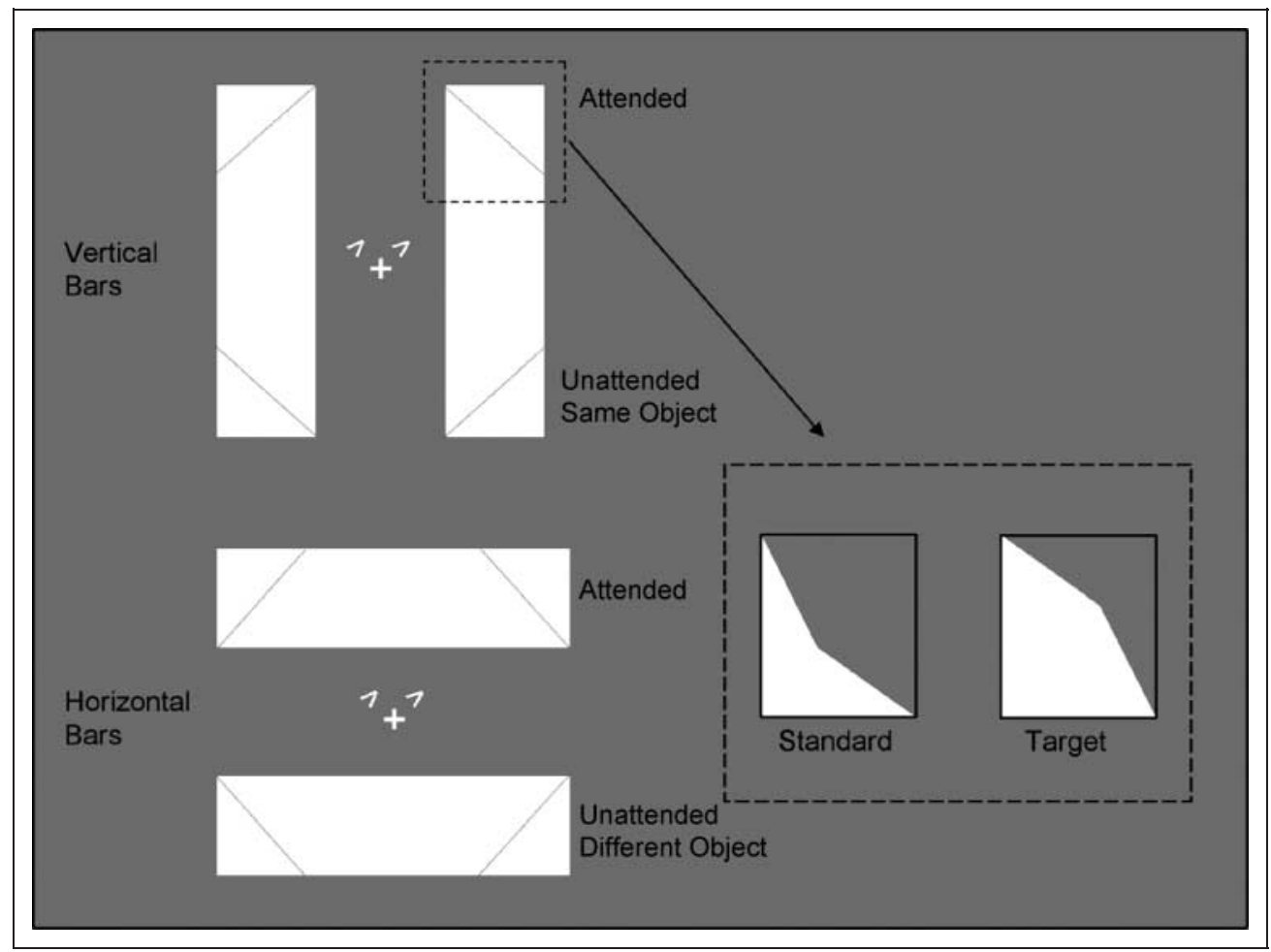

brain activity elicited by unattended corner offsets when these formed part of the attended object versus when they belonged to a different, unattended object.

\section{ERP Attention Effects}

As in previous studies, the effects of spatial attention were evident as amplitude modulations of the early, sensory-evoked P1 (80-128 msec poststimulus onset) and N1 (160-196 msec) components (Figure 2). For each quadrant, attended stimuli elicited significantly larger P1 and N1 amplitudes than did the same stimuli when unattended. In general, these components were largest over the contralateral scalp (Table 1).

Object-selective amplitude modulations were also evident in the N1 component for each quadrant (Figure 2). In particular, the $\mathrm{N} 1$ amplitudes elicited by unattended stimuli were larger when they formed part of the attended object versus when they belonged to a different object. As was the case for the spatial attention effect on N1, the object-related N1 effect was generally largest over the contralateral hemisphere (Table 2). The objectbased attention effect on N1 was significantly smaller than the spatial attention effect $(p<.05$ for all quadrants). Object-based selection did not significantly modulate the P1 component ( $p>.05$ for all quadrants).

The scalp topographies of the N1 difference waves associated with spatial attention and object-selective attention were very similar to one another, with maximum amplitudes over the posterior contralateral scalp (Figure 2). Although these topographies differed accord- ing to the visual quadrant of the eliciting stimulus, comparisons of the spatial and object difference topographies over the posterior scalp using the method of McCarthy and Wood (1985) did not reach significance for any quadrant (all $p s>.05$ ).

\section{Source Localization}

To estimate the neuroanatomical sources of these attention-related ERP modulations, inverse dipole modeling of the spatial- and object-selective difference topographies was carried out using the Brain Electrical Source Analysis (BESA) algorithm. Pairs of symmetrical dipoles were fit to the difference topographies during the interval of the P1 (80-128 msec, for spatial attention only) and N1 (160-196 msec) components. For each quadrant, a pair of symmetrical dipoles in the ventrolateral occipital cortex could account for the amplitude modulation of the P1 component in the spatial attention difference waves (see Table 3 for dipole coordinates). The locations of these dipoles correspond closely to previously reported ventral sources of the P1 (Di Russo, Martinez, \& Hillyard, 2003; Di Russo, Sereno, Pitzalis, \& Hillyard, 2002; Martinez et al., 1999). The subsequent N1 components in these spatial attention difference waves were well fit by pairs of symmetrical dipoles situated more posteriorly in the ventrolateral occipital cortex. The N1 components in the object-selective difference waves were accounted for by similarly situated dipole pairs in the ventrolateral occipital cortex (Table 3). These dipole models of the 
Figure 2. Grand-averaged ERPs to stimuli in each quadrant when attended (yellow waveforms) and unattended (red and green waveforms). ERPs to unattended stimuli belonging to the same (attended) object are shown as red waveforms. ERPs to unattended stimuli forming part of a different (unattended) object are shown as green waveforms. ERPs shown are from contralateral parietal electrode sites (P1/P2 and $\mathrm{CP} 1 / \mathrm{CP} 2)$. Voltage maps of the attention-related difference waves associated with spatial attention (yellow minus green waveforms) and object attention (red minus green waveforms) are shown for each quadrant in the N1 latency range (160-196 msec). Voltage scale on the left applies to all spatial attention maps. Voltage scale on the right applies to all object attention maps.

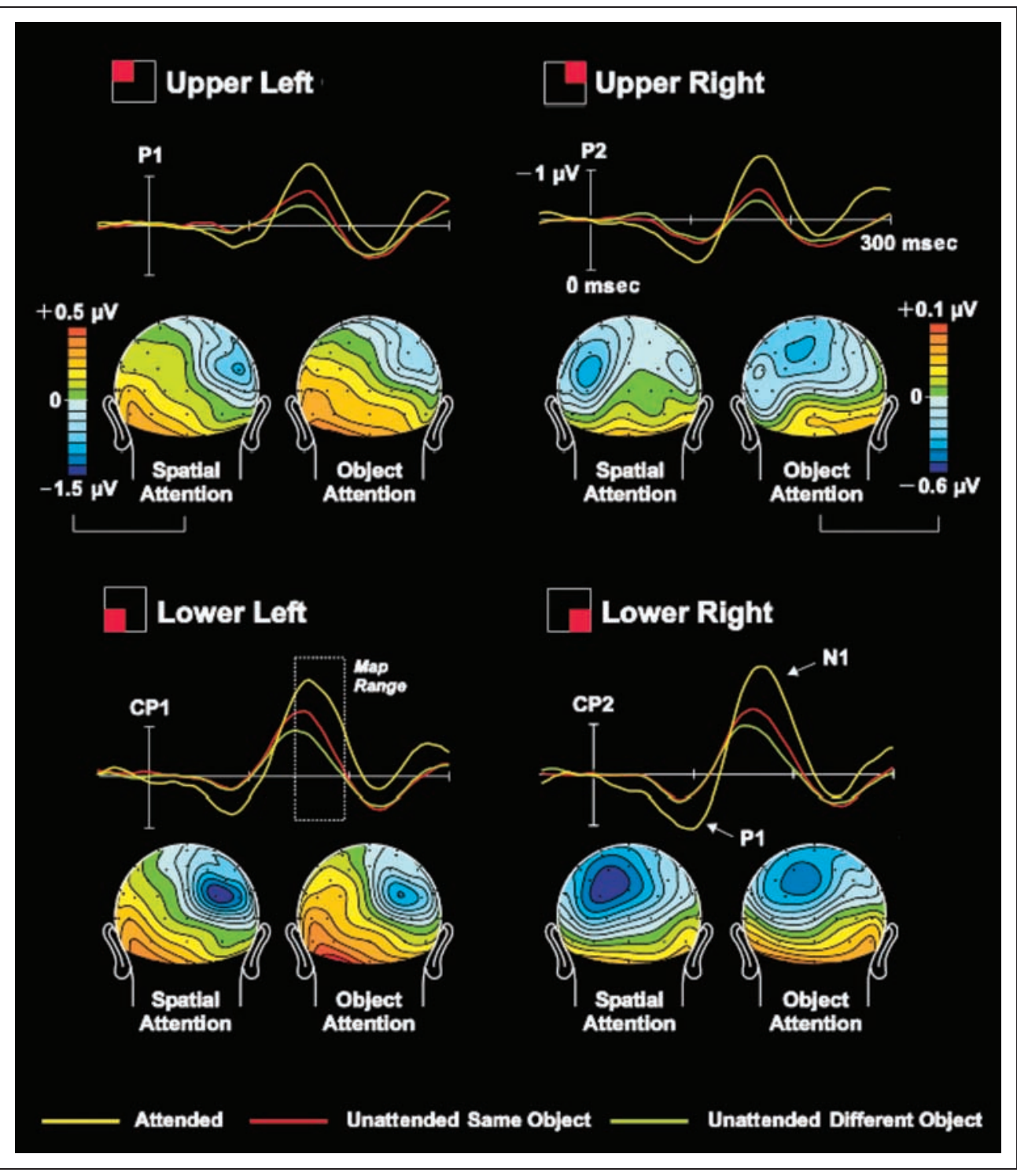

spatial- and object-selective N1 modulations all accounted for more than $90 \%$ of the variance in scalp voltage topography for each quadrant over the time range 100-196 msec.

\section{fMRI Attention Effects}

As with the ERPs, spatial attention effects were assessed for each quadrant by comparing the BOLD signal during blocks when that quadrant was attended to the signal elicited when the same quadrant was unattended. These comparisons were restricted to regions of interest (ROIs) defined for each quadrant during passive stimulation (see Methods). As in previous studies of spatial attention (Di Russo et al., 2003; Martinez et al., 2001; Brefczynski \& DeYoe, 1999; Tootell et al., 1998), analyses of individual subject data as well as subsequent group analyses resulted in significant attention-related en- hancements of the BOLD signal within the retinotopic visual areas of the contralateral hemisphere. For the 28 experimental conditions $(7$ subjects $\times 4$ attended quadrants), the different retinotopic areas showed significantly enhanced activity with spatial attention in the following percentages of the conditions: V1 (64\%), V2 (64\%), V3/VP (90\%), V3A (100\%), V4v (100\%).

Analyses of object-selective attention effects were restricted to activations occurring within ROIs representing unattended spatial locations. Depending on the orientation of the bars, these unattended locations formed part of the object being attended or belonged to the other object. In the individual subjects' analyses, object-selective attention produced less extensive enhancements of activity in the retinotopic areas than did spatial attention: V1 (4\%), V2 (0\%), V3/VP (18\%), V3A (36\%), V4v (43\%). These activations were significantly less than for spatial attention in the early visual areas 
Table 1. Effects of Spatial Attention on ERP Amplitudes

\begin{tabular}{|c|c|c|c|c|c|c|c|c|c|c|c|c|c|}
\hline \multirow[b]{2}{*}{ Quadrant } & \multirow[b]{2}{*}{ Hemisphere } & \multicolumn{2}{|c|}{$\begin{array}{c}\text { P1 } \\
\text { Amplitudes }\end{array}$} & \multicolumn{2}{|c|}{$\begin{array}{c}\text { Attention } \\
\text { (Attended vs. } \\
\text { Unattended) }\end{array}$} & \multicolumn{2}{|c|}{$\begin{array}{l}\text { Hemisphere } \\
\text { (lpsilateral vs. } \\
\text { Contralateral) }\end{array}$} & \multicolumn{2}{|c|}{$\begin{array}{c}\text { N1 } \\
\text { Amplitudes }\end{array}$} & \multicolumn{2}{|c|}{$\begin{array}{l}\text { Attention } \\
\text { (Attended vs. } \\
\text { Unattended) }\end{array}$} & \multicolumn{2}{|c|}{$\begin{array}{l}\text { Hemisphere } \\
\text { (lpsilateral vs. } \\
\text { Contralateral) }\end{array}$} \\
\hline & & Attended & Unattended & $F(1,18)$ & $p$ & $F(1,18)$ & $p$ & Attended & Unattended & $F(1,18)$ & $p$ & $F(1,18)$ & $p$ \\
\hline \multirow[t]{2}{*}{ UL } & $\mathrm{LH}$ & 0.22 & 0.00 & 7.05 & $<.016$ & 13.18 & $<.002$ & -0.41 & -0.27 & 9.96 & $<.005$ & 25.76 & $<.001$ \\
\hline & $\mathrm{RH}$ & 0.52 & 0.18 & & & & & -1.05 & -0.50 & & & & \\
\hline \multirow[t]{2}{*}{ UR } & $\mathrm{LH}$ & 0.69 & 0.34 & 11.64 & $<.003$ & 18.68 & $<.001$ & -0.86 & -0.35 & 13.10 & $<.002$ & 2.12 & $n s$ \\
\hline & $\mathrm{RH}$ & 0.33 & 0.10 & & & & & -0.63 & -0.31 & & & & \\
\hline \multirow[t]{2}{*}{ LL } & $\mathrm{LH}$ & 0.36 & 0.03 & 16.70 & $<.001$ & 1.87 & ns & -0.88 & -0.39 & 60.09 & $<.001$ & 39.40 & $<.001$ \\
\hline & $\mathrm{RH}$ & 0.62 & 0.02 & & & & & -1.92 & -0.72 & & & & \\
\hline \multirow[t]{2}{*}{ LR } & $\mathrm{LH}$ & 0.61 & 0.22 & 10.41 & $<.005$ & 2.27 & ns & -1.66 & -0.56 & 55.89 & $<.001$ & 6.90 & $<.01$ \\
\hline & $\mathrm{RH}$ & 0.46 & 0.12 & & & & & -1.21 & -0.40 & & & & \\
\hline
\end{tabular}

P1 and N1 amplitudes elicited by stimuli in each quadrant are given as mean voltages (in microvolts) at electrodes over the left (LH) and right (RH) hemispheres over latency windows used in the ANOVAs.

$\left(\mathrm{V} 1, \mathrm{~V} 2\right.$, and V3/VP) $\left(\chi^{2}>15.0, p<.001\right)$. Group analysis of object-selective attention effects showed enhanced BOLD signals within multiple regions of the extrastriate cortex including the middle occipital gyrus, fusiform gyrus, and parts of the superior and inferior parietal lobes (Table 4). A conjunction analysis was carried out to identify areas activated in common by spatial- and object-selective attention. Although these overlapping regions varied slightly among the four quadrants, a contralateral region in lateral occipital cortex, in or near the middle occipital gyrus (Brodmann's area [BA] 18/19), was activated during both spatial and object attention in all quadrants (Figure 3A, Table 4).
To compare the anatomical sources of the attentionrelated N1 modulations and fMRI activations, the coordinates of the $\mathrm{N} 1$ dipoles were transformed into a common reference frame and superimposed on the group activation maps. As shown in Figure 3B, the dipoles accounting for both object- and spatial-selective N1 modulations were situated very close to a zone of activation in the middle occipital gyrus, in or near a region that has been described as the lateral occipital complex (LOC) (Lerner, Hendler, \& Malach, 2002; Grill-Spector, Kourtzi, \& Kanwisher, 2001; Malach et al., 1995). In a further analysis, pairs of dipole sources were "seeded" to the locations of the LOC activation sites in each quadrant as identified in the group analysis. For

Table 2. Effects of Object-Selective Attention on N1 Amplitudes

\begin{tabular}{|c|c|c|c|c|c|c|c|}
\hline \multirow[b]{2}{*}{ Quadrant } & \multirow[b]{2}{*}{ Hemisphere } & \multicolumn{2}{|c|}{ N1 Amplitudes } & \multicolumn{2}{|c|}{$\begin{array}{c}\text { Attention (Same vs. } \\
\text { Different Object) }\end{array}$} & \multicolumn{2}{|c|}{$\begin{array}{c}\text { Hemisphere (Ipsilateral } \\
\text { vs. Contralateral) }\end{array}$} \\
\hline & & Same & Different & $F(1,18)$ & $p$ & $F(1,18)$ & $p$ \\
\hline \multirow[t]{2}{*}{ UL } & LH & -0.38 & -0.27 & 4.09 & $<.048$ & 21.74 & $<.001$ \\
\hline & $\mathrm{RH}$ & -0.71 & -0.50 & & & & \\
\hline \multirow[t]{2}{*}{ UR } & LH & -0.49 & -0.35 & 5.27 & $<.034$ & 0.443 & $n s$ \\
\hline & $\mathrm{RH}$ & -0.43 & -0.31 & & & & \\
\hline \multirow[t]{2}{*}{ LL } & LH & -0.52 & -0.39 & 8.62 & $<.009$ & 33.03 & $<.001$ \\
\hline & $\mathrm{RH}$ & -1.02 & -0.72 & & & & \\
\hline \multirow[t]{2}{*}{ LR } & LH & -0.86 & -0.56 & 17.70 & $<.001$ & 4.06 & $<.049$ \\
\hline & $\mathrm{RH}$ & -0.60 & -0.40 & & & & \\
\hline
\end{tabular}

N1 amplitudes elicited by unattended stimuli in each quadrant are given as mean voltage (in microvolts) at electrodes over LH and RH in latency window used in the ANOVA. The main effect of attention is the comparison of amplitudes elicited by stimuli belonging to the attended (Same) versus unattended (Different) object. 
Table 3. Talairach Coordinates of Dipoles and Residual Variances (RV) of Dipole Models Fit to the Attentional Difference Waves for Spatial Attention and Object Attention

\begin{tabular}{|c|c|c|c|c|c|c|c|c|c|c|c|}
\hline \multirow[b]{2}{*}{ Quadrant } & \multirow{2}{*}{$\begin{array}{c}\text { Spatial Attention } \\
(R V, \%)\end{array}$} & \multicolumn{3}{|c|}{ P1 } & \multicolumn{3}{|c|}{ N1 } & \multirow{2}{*}{$\begin{array}{c}\text { Object Attention } \\
(R V, \%)\end{array}$} & \multicolumn{3}{|c|}{$N 1$} \\
\hline & & $x$ & $y$ & $z$ & $x$ & $y$ & $z$ & & $x$ & $y$ & $z$ \\
\hline UL & 7 & \pm 44 & -62 & -13 & \pm 43 & -70 & -3 & 10 & \pm 40 & -65 & -5 \\
\hline UR & 7 & \pm 42 & -61 & -13 & \pm 38 & -70 & 0 & 9 & \pm 30 & -75 & -5 \\
\hline LL & 3 & \pm 49 & -65 & -17 & \pm 31 & -75 & -2 & 7 & \pm 33 & -72 & -3 \\
\hline LR & 4 & \pm 41 & -66 & -9 & \pm 33 & -72 & -3 & 8 & \pm 40 & -71 & -4 \\
\hline
\end{tabular}

Values are in millimeters.

each quadrant, the ERP difference topographies for the object-selective attention effect were fit by these location-constrained dipoles. The residual variance between these constrained models and the difference topographies ranged from $9 \%$ to $14 \%$. Thus, the objectselective N1 modulation was well accounted for in both constrained and unconstrained dipole models, consistent with a generator source in the LOC region.

\section{DISCUSSION}

The ERP data reported here are in agreement with the previous findings of He et al. (2004) that the allocation of spatial attention facilitates the sensory processing not only of stimuli at the attended location but also of more distant stimuli occurring within the boundaries of an object that encompasses the attended location. In both studies, this object-selective effect was reflected in increased amplitude of the posterior, sensory-evoked N1 component over the interval 150-190 msec in response to uncued stimuli included within the common object. There is considerable evidence that this posteriorly distributed N1 component reflects the discriminative processing of visual information in the extrastriate cortex (Hopf, Vogel, Woodman, Heinze, \& Luck, 2002; Vogel \& Luck, 2000; Ritter, Simson, \& Vaughan, 1988)

Table 4. Talairach Coordinates of Brain Areas Showing Significant Activation in Group $t$ Tests Comparing Activity in Unattended ROIs at Each Quadrant When Attention Was Focused on the Same Versus Different Object (Left) and Areas Identified in the Conjunction Analysis Showing Significant Activation during Both Object and Spatial Attention Conditions (Right)

\begin{tabular}{|c|c|c|c|c|c|c|c|c|}
\hline & Object Attention Effects & $x$ & $y$ & $z$ & Conjunction Effects & $x$ & $y$ & $z$ \\
\hline \multirow[t]{4}{*}{ Upper left quadrant } & R. mid. occ. (BA 18) & 42 & -78 & -5 & R. mid. occ. (BA 18) & 49 & -70 & -4 \\
\hline & R. fusiform (BA 37) & 34 & -58 & -16 & R. fusiform (BA 37) & 30 & -58 & -17 \\
\hline & R. mid. temp. (BA 22) & 51 & -46 & 9 & & & & \\
\hline & R. inf. par. (BA 40) & 53 & -40 & 25 & & & & \\
\hline \multirow[t]{4}{*}{ Upper right quadrant } & L. ling. & -15 & -70 & -12 & L. ling. & -16 & -68 & -10 \\
\hline & L. mid occ. (BA 19) & -48 & -72 & 2 & L. mid. occ. (BA 19) & -44 & -73 & 0 \\
\hline & L. inf. par. (BA 7) & -38 & -70 & 47 & L. inf. par. (BA 7) & -42 & -66 & 50 \\
\hline & R. inf. par. (BA 40) & 55 & -46 & 26 & & & & \\
\hline \multirow[t]{3}{*}{ Lower left quadrant } & R. mid. occ. & 34 & -75 & 0 & R. mid. occ. (BA 19) & 38 & -71 & 0 \\
\hline & R. precuneus (BA 19) & 30 & -70 & 39 & & & & \\
\hline & R. sup. par. (BA 7) & 27 & -58 & 44 & & & & \\
\hline \multirow[t]{4}{*}{ Lower right quadrant } & L. mid. occ. (BA 18) & -22 & -91 & 6 & L. mid. occ. (BA 18) & -23 & -91 & 9 \\
\hline & L. cuneus (BA 18) & -7 & -86 & 15 & L. cuneus (BA 18) & -6 & -86 & 14 \\
\hline & L. mid. occ. (BA 19) & -34 & -77 & -3 & L. mid. occ. (BA 19) & -36 & -76 & -1 \\
\hline & L. sup. par. (BA 7) & -20 & -62 & 44 & & & & \\
\hline
\end{tabular}

Values are in millimeters.

R. mid. occ. = right middle occipital; R. fusiform = right fusiform; R. mid. temp. = right middle temporal; $\mathrm{R}$. inf. par. = right inferior parietal; L. ling. = left lingual; L. mid. occ. = left middle occipital; L. inf. par. = left inferior parietal; R. inf. par. = right inferior parietal; R. precuneus $=$ right precuneus; R. sup. par. = right superior parietal; L. cuneus = left cuneus; L. sup. par. = left superior parietal. 
Figure 3. (A) fMRI activations for spatial (left), objectselective attention (center), and results of conjunction analysis (right) for a single subject in the study. Dotted white lines depict the boundaries of retinotopically organized visual areas. Data from each quadrant are shown in separate rows. For each quadrant, only the contralateral (flattened) hemisphere is shown. In all quadrants, spatial attention resulted in widespread activations throughout striate and extrastriate cortex. Activations related to objectselective attention were less extensive but, in all quadrants, included a region in the lateral occipital cortex that coincided with an area activated during spatial attention. Color bar on lower left depicts significance values $(p)$ of the data shown, only areas with $p<.05$ are

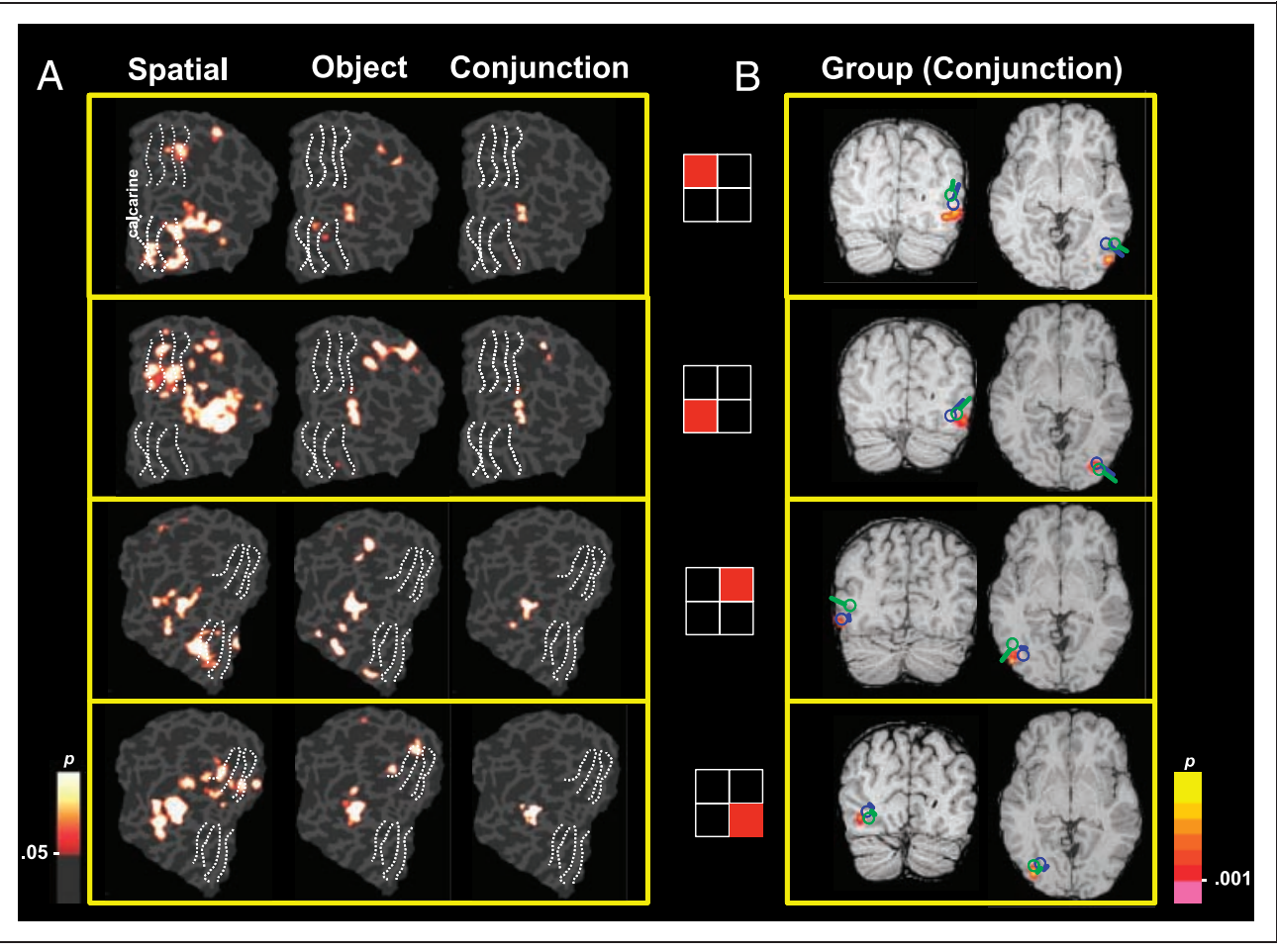
shown. (B) Spatial correspondence between areas activated in common by spatial and object attention and N1 dipole models. fMRI activations resulting from the group conjunction analysis are shown superimposed on the corresponding MRI slices from a single subject. In all quadrants, an area corresponding to the LOC region was significantly activated during both conditions. This area corresponds well with the locations of the N1 dipoles fit to the object (blue) and spatial (green) grand-averaged attentional difference waves. Only the contralateral member of each symmetrical dipole pair is shown. Color bar on lower right depicts significance values $(p)$ of the data shown; only areas with $p<.001$ are shown.

and that its amplitude can be enhanced by spatially focusing attention on the stimulus location (Hopfinger, Luck, \& Hillyard, 2004; Di Russo et al., 2003; Mangun, 1995; Luck et al., 1994). The present results and those of He et al. thus support the hypothesis that directing spatial attention to one part of an object results in the facilitation of discriminative sensory processing of the entire object, although the degree of facilitation is considerably reduced for those portions of the object more distant from the attended location.

In their design, He et al. (2004) used an exogenous cue flashed at the most probable target location to direct spatial attention. By varying the percent validity of this cue, they distinguished between a voluntary allocation of attention that was dependent on high cue validity and a reflexive/automatic allocation that was not. Their behavioral results led He et al. to suggest that visual objects were selected (at an early sensory level) by the reflexive rather than the voluntary mechanism. The present ERP results, however, indicate that a purely voluntary allocation of spatial attention can also produce object-based sensory enhancement in the visual cortical pathways. The spatiotemporal pattern of object-selective N1 enhancement observed here was very similar to that described by He et al., despite significant differences between the two studies. For example, whereas the present design used voluntary/endogenous cuing, con- tinuous stimulus sequences, offset stimuli, and a difficult discrimination, He et al. employed reflexive, trial-by-trial cuing and simple detection of onset targets. Similarly distributed posterior N1 components have also been reported to reflect object-selective attention in very different types of tasks in which subjects attended to one of two superimposed transparent surfaces formed by rotating dot arrays (Lopez, Rodriguez, \& Valdes-Sosa, 2004; Valdes Sosa et al., 2003; Pinilla, Cobo, Torres, \& Valdes-Sosa, 2001), to superimposed geometric figures (Weber et al., 1997), or to line segments during performance of the line-bisection task (Foxe, McCourt, \& Javitt, 2003). Taken together, these studies suggest that the posterior N1 component may reflect a stage of processing at which unified perceptual objects are encoded and may be selectively highlighted by attention.

In the present study we compared the spatiotemporal patterns of ERP modulation associated with spatialselective and object-selective attention within the same experiment. This analysis suggests that the mechanisms for spatial and object selection overlap substantially, but are not identical. Whereas spatial selection was associated with enlarged P1 and N1 components to attendedlocation stimuli, in agreement with previous studies (reviewed in Di Russo et al., 2003), the object-selective effect was reflected only by an increase in the N1. Previous studies have ascribed separate and dissociable 
roles for the P1 and N1 components in spatial attention, with P1 indexing an early stage of suppressing irrelevant inputs and $\mathrm{N} 1$ a subsequent stage of facilitated discriminative processing of relevant inputs (Luck et al., 1994; reviewed in Hopfinger et al., 2004). The present findings suggest that the allocation of spatial attention includes an object-selective component that acts at the higher, discriminative stage reflected in the N1. This proposal is in line with the hypothesis that object-based selection may be mediated by spatial attention through a mechanism of object-guided spatial selection (Weber et al., 1997).

The ERP findings reported here complement the object-selective fMRI effects reported in an Egly-style task by Müller and Kleinschmidt (2003). They observed anticipatory, object-selective fMRI activations in early visual areas during the cue-target interval, which were taken as evidence that the deployment of attention in space is guided by the presence of objects. Posttarget activations were also observed, which were significantly larger (when averaged over all the early visual areas) in cortical zones corresponding to the uncued end of the cued object than to zones corresponding to an equidistant end of an uncued object. Due to the low time resolution of fMRI, however, it is difficult to determine (1) whether these posttarget activations reflected immediate sensory processing of the target information as opposed to a more prolonged attentional control process or (2) at what level of the visual cortical pathways posttarget object-selective processing was first initiated. The ERP results of the present study (and those of He et al., 2004) provide critical evidence that the object-selective effect in the Egly paradigm does in fact involve a facilitation of sensory processing of stimuli belonging to unattended or uncued regions of an attended object.

In the current study, dipole modeling of the objectselective N1 modulation indicated a principal source in lateral occipital cortex, coinciding with fMRI activation in the LOC region. Whereas multiple sources in ventral and lateral occipital cortex reportedly contribute to the posterior N1 component (Di Russo et al., 2003; Di Russo et al., 2002), recent studies have reported enhanced N1 components associated with object perception with similar cortical sources in the LOC region (Murray, Foxe, Javitt, \& Foxe, 2004; Rose, Schmid, Winzen, Sommer, \& Buchel, 2004; Murray et al., 2002; Foxe, Murray, \& Javitt, 2005). In particular, a series of studies conducted by Murray et al. (2002) identified a parieto-occipital N1 component (peak latency of $146 \mathrm{msec}$, poststimulus) that was modulated by the presence of objects defined by illusory contours. Using a combination of ERP recordings, source localization analyses, and fMRI, this objectselective N1 component was associated with enhanced activity in the LOC region regardless of the shape or retinotopic position of the object. Taken together, our findings and those of Murray et al. are consistent with numerous experiments reporting enhanced fMRI signals in LOC during object recognition processes (Lerner et al., 2002; Grill-Spector et al., 2001; Grill-Spector et al., 1999; Grill-Spector, Kushnir, Edelman, Itzchak, \& Malach, 1998; Grill-Spector, Kushnir, Hendler, et al., 1998; Malach et al., 1995), which support the hypothesis that the LOC region plays a major role in object perception and recognition (see also Doniger et al., 2001; Doniger et al., 2000).

The two major hypotheses put forward to account for the same-object advantage in cued attention tasks have been characterized as "sensory modulation" on the one hand and "priority setting" on the other (Shomstein \& Yantis 2002). The present physiological evidence appears incompatible with priority setting models that attribute object-specific benefits to an "attentional prioritization ... that affects the order in which different regions of the scene are visually investigated when multiple attentional 'glimpses' are required." Such a prioritized visual search was hypothesized to occur in trial-by-trial cuing tasks when relevant stimuli appear at uncued locations on invalid trials Based on evidence from such tasks, Shomstein and Yantis (2002) concluded that "when attention is highly focused on a single location ... object-based prioritization cannot exert an influence, and object-based effects are not observed." The present experiment, however, did obtain objectbased ERP and fMRI modulations in a design where attention was highly focused upon only one location (as indicated by high levels of target discrimination accuracy) and all other locations were irrelevant. Under these conditions, a prioritized search of the irrelevant locations was not required and thus could not account for the object-selective modulations of ERPs and fMRI activations that were observed.

In conclusion, the present ERP results provide direct physiological evidence that the deployment of spatial attention to one part of an object results in a facilitation of sensory processing of the entire object, even those parts that are not relevant to the current task. This object-selective sensory modulation was reflected in amplitude enhancement of the N1 component, which had the same timing, polarity, and source localization as the N1 enhancement associated with spatial attention when ERPs to attended versus unattended locations were compared. This spatiotemporal equivalence suggests that spatial attention not only facilitates the processing of stimuli at an attended location (reflected in amplification of P1 and N1 components) but also engages an object-selective mechanism (reflected in amplification of the N1) that strengthens the sensory representations of entire objects that encompass the attended location. This object-based facilitation takes place in or near an extrastriate cortical region (LOC) that has been widely implicated in object perception and may reinforce the perceptual integrity of objects at or near the focus of attention. 


\section{METHODS}

\section{Task and Stimuli}

Nineteen subjects ( 8 women, mean age 20 years) participated in the ERP portion of the study; an additional 17 subjects (9 women, mean age 24 years) took part in the fMRI experiment. The task and stimuli were the same for both the electrophysiological recording and fMRI scanning sessions. Stimuli were pairs of horizontal or vertical bars (white on a gray background) measuring $4^{\circ}$ of visual angle from a central fixation point to each corner (Figure 1). While the bars were continuously displayed, the task stimuli consisted of brief (100 msec) offsets of the bars' corners, leaving either a white concave edge (standards, $p=.8$ ) or convex edge (targets, $p=.2$ ). Corner offsets occurred one at a time in random order in the different quadrants at intervals of 400600 msec during 20-sec runs. A different randomized sequence of quadrant offsets was used in each run. Runs with horizontal bars or vertical bars were given in randomized, counterbalanced order.

During each run, subjects were instructed to maintain fixation on a central cross while covertly directing attention to the corner (quadrant) indicated by a pair of arrows presented just above or below fixation. During half the blocks (of eight runs each) the cues alternated between upper left (UL) and upper right (UR) quadrants, and in the remaining half they alternated between lower left (LL) and lower right (LR) quadrants. A total of 20 runs were given in each of the eight experimental conditions ( 4 quadrants attended $\times 2$ bar orientations).

To localize fMRI activation sites with respect to retinotopic visual areas, seven of the subjects who participated in the fMRI experiment returned for a second scanning session during which two scans measuring eccentricity (dilating/expanding concentric rings) and two scans measuring polar angle (rotating wedge) were acquired according to the methods described in Sereno et al. (1995). The cortical surface of these subjects was rendered and flattened and the resulting retinotopic data containing the boundaries of each visual area was projected onto each hemisphere.

\section{Electrophysiological Recordings and Data Analysis}

Subjects sat in a dimly lit recording chamber while viewing stimuli presented on a video monitor. The electroencephalogram (EEG) was recorded with SA amplifiers (San Diego, CA) from 64 electrode sites (Electro-Cap International, Eaton, $\mathrm{OH}$ ) using a modified 10-20 system montage (Di Russo et al., 2003; Nuwer et al., 1998). During recording, the right mastoid served as reference and the left mastoid was an active electrode. Scalp channels were algebraically re-referenced off-line to the averaged mastoids. Horizontal eye movements were monitored via electrodes at the left and right outer canthi. Blinks were recorded with an electrode below the left eye.

The EEG was digitized at $250 \mathrm{~Hz}$ with an amplifier band pass of 0.01 to $80 \mathrm{~Hz}$. Computerized artifact rejection was performed prior to signal averaging in order to discard epochs in which deviations in eye position, blinks, or amplifier blocking occurred. On average, 9\% of the trials were rejected due to a combination of these artifact sources.

Time-locked ERPs to corner-offset stimuli (standards only) were grand-averaged across all subjects according to the quadrant of presentation (UL, LL, UR, and LR), whether they were attended or unattended, and stimulus configuration (vertical or horizontal bars). To assess effects of spatial attention, ERPs to an attended stimulus (averaged over conditions with vertical and horizontal bars) were compared to the ERPs elicited by the same stimulus when it was unattended and was not at the other end of the attended bar. For example, the unattended ERP to an UR stimulus was formed by averaging the ERP elicited by a UR corner offset when attention was focused on the UL quadrant and the bars were oriented vertically together with the ERP elicited by the same stimulus during blocks when attention was directed toward the LR quadrant and the bars were horizontal. Attended and unattended averaged ERPs were generated for stimuli in each quadrant. Approximately 250-300 stimulus events (per subject) were included in each averaged waveform. Scalp topography maps and source localization analyses for the spatial attention effect in each quadrant were based on attentional difference waves formed by subtracting the averaged unattended from the averaged attended waveforms.

Object attention effects were quantified by comparing the amplitudes of ERPs to unattended stimuli only as a function of whether these stimuli belonged to the attended object (same-object condition) or the unattended object (different-object condition). As in the spatial attention analyses, these comparisons were also averaged over stimulus configurations with horizontal and vertical bars. For example, ERPs in the same-object condition for the UR quadrant was formed by averaging the ERP elicited by the UR corner offsets when attention was directed to the UL quadrant and the bars were horizontal together with the ERP elicited by the stimuli when attention was focused on the LR quadrant and the bars were vertically oriented. In the different-object condition for the UR quadrant, the ERP elicited during attention to the UL quadrant with vertical bars was averaged together with the ERP elicited during attention to the LR quadrant with horizontal bars. Same-object and different-object averages were generated in this way for stimuli in each quadrant. Object-selective attention was evidenced in the difference wave formed by subtracting the averaged unattended different-object ERP from the averaged unattended same-object ERP. These object-selective difference waves were used in 
subsequent source localization analyses and scalp topography maps. Corner offsets in the quadrant diagonal to the attended quadrant were not included in any of these analyses.

To assess the significance of attention effects, the prominent ERP components P1 and N1 were measured as mean amplitudes averaged across the 10 posterior electrode sites in each hemisphere where these components were largest. Separate analyses were carried out for each quadrant. Mean amplitudes of the P1 (over 80$128 \mathrm{msec}$ ) and N1 (over 160-196 msec) components with respect to a 100-msec prestimulus baseline were subjected to repeated-measures analysis of variance (ANOVA) with factors of Attention (for spatial attention, same stimulus when attended vs. unattended as described above; for object attention, same unattended stimulus when attention was focused on the same vs. different object as described above) and Hemisphere (ipsilateral vs. contralateral to the eliciting stimulus). These time windows for measuring P1 and N1 were chosen because they encompassed the attention-related amplitude modulations that were stable in scalp topography within their respective windows. The scalp distributions of the N1 amplitude modulations produced by spatial- and object-selective attention were compared across the entire array of electrodes after normalizing their amplitudes prior to ANOVA using the method recommended by McCarthy and Wood (1985).

\section{Modeling of ERP Sources}

Estimation of the dipolar sources of the N1 modulations produced by spatial- and object-selective attention was carried out using the BESA software (version 5.0). Pairs of dipoles, constrained to be symmetrical in location but allowed to vary freely in orientation, were fit sequentially over specific latency ranges. Modeling of the N1 modulation with spatial attention was carried out for each quadrant on the attentional difference waves described above. The early phase of this difference wave corresponding to the P1 component was fit over 80120 msec with a pair of symmetrical dipoles, which were then fixed in location and orientation before fitting the N1 over 160-196 msec with a second pair of mirrorsymmetrical dipoles. Dipole fits were only accepted if they accounted for $90 \%$ or more of the variance in scalp topography and could be reproduced using several different starting locations.

The N1 modulation with object-selective attention was modeled for each quadrant from the object-attention difference waves described above. As in the spatial attention models, a pair of mirror-symmetrical dipoles was fit in the N1 latency range of 160-196 msec (there were no significant earlier components in this difference wave).

In order to estimate the positions of the dipole sources with respect to brain anatomy and fMRI activa- tions, the dipole coordinates calculated from the group average ERP distributions were transformed into the standardized coordinate system of Talairach and Tournoux (1988) and projected onto the structural MRI of a single subject along with the group-averaged fMRI activations.

\section{fMRI Experiment}

The stimuli and task were identical to that used in the ERP study with the addition of a passive stimulation condition that was used to define functional ROIs activated by corner-offset stimuli in each quadrant. Each participant took part in six scans, the first two of which were passive with no task involved. During the passive scans, corner-offset stimuli were delivered to one quadrant at a time during 20-sec runs. In the remaining four scans, stimuli were delivered randomly to each quadrant, and during successive 20-sec runs subjects were cued to alternate attention between the left and right quadrants in either the upper or lower visual field. The stimulus configuration consisted of vertical bars for half the runs and horizontal bars for the remaining half. Each scan consisted of 16 runs (passive stimulation of or attention to each quadrant occurred four times per scan, twice with vertical bars and twice with horizontal bars).

\section{Image Acquisition}

T2*-weighted echo-planar images (EPIs) (repetition time $=2 \mathrm{sec}$, echo time $=40 \mathrm{msec}$, flip angle $=90^{\circ}$; voxel size $=3 \mathrm{~mm}^{3}$; matrix size $64 \times 64$ ) were acquired on a 3-T SMIS (SMIS/Marconi, Highland Heights, $\mathrm{OH}$ ) system equipped with a head volume coil. During each scan, 164 volumes were acquired on each of 20 contiguous slices in the coronal plane beginning at the occipital pole. The first four volumes were discarded prior to all analyses to allow for stabilization of the BOLD signal. Visual stimulation was delivered through MR-compatible liquid crystal display goggles (Resonance Technology Inc., Northridge, CA). For anatomical localization of functional data, high-resolution $\left(1 \times 1 \times 1 \mathrm{~mm}^{3}\right)$ images of the entire brain were acquired from each subject using a standard MPRAGE sequence. A geometrical representation of the cortical surface was generated for seven subjects using the procedures incorporated in the software package FreeSurfer (Dale et al., 2000; Fischl, Sereno, \& Dale, 1999; Fischl, Sereno, Tootell, \& Dale, 1999; Dale \& Sereno, 1993).

\section{Data Preprocessing}

The fMRI data were analyzed with the AFNI software package (Cox, 1996). Prior to statistical testing the EPI images from individual subjects were realigned to the first included volume (motion never exceeded $0.85 \mathrm{~mm}$ 
along any axis), linearly detrended, and slice time corrected. Functional images were coregistered with the high-resolution anatomical images and projected into Talairach coordinate space before being spatially smoothed with a Gaussian kernel of $6 \mathrm{~mm}$ full width at half maximum (FWHM). The statistical significance levels and minimum cluster size of activation maps were calculated using a Monte Carlo simulation (Alpha Sim, part of the AFNI package). Group data are reported only for voxels with $t$ values greater than $3.60(p<.001)$ that belonged to clusters of eight or more neighboring voxels $\left(>216 \mathrm{~mm}^{3}\right)$. Individual subject data from the regression analyses (below) was thresholded at $p<.05$ and only included voxels neighbored by four or more voxels with statistically significant values.

\section{Passive Stimulation-Defining ROI Masks}

To estimate the BOLD response associated with passive stimulation in each visual quadrant, regressors representing the timing of each stimulation epoch were convolved with a canonical hemodynamic response function and entered in a multiple regression analysis. General linear tests contrasting stimulation at each quadrant versus the remaining quadrants were conducted on each individual subject's data. These singlesubject maps were entered into a groupwise $t$ test comparing the activation pattern resulting from each linear contrast to the null hypothesis. The resulting four statistical maps were used as ROI masks and all subsequent analyses were restricted to voxels within these ROIs.

\section{Attention Scans}

For each subject, attention-related BOLD responses within the ROIs representing each quadrant were calculated by use of a multiple regression analysis with eight regressors (attend UL, UR, LL, and LR quadrants, for vertical and horizontal bars). Individual maps of the regressor coefficients associated with attention to each quadrant were entered into separate between-subjects paired $t$ tests. Spatial attention effects were assessed by comparing activation patterns for each quadrant when attended (averaged over horizontal and vertical bar conditions) versus when unattended and not belonging to the attended object (see ERP, Methods, for an example). Analyses of object-selective attention effects were conducted by comparing the regressor coefficient maps within each quadrant's ROI when unattended as a function of whether the attended quadrant belonged to the attended (same) bar versus the unattended (different) bar (see, e.g., ERP, Methods).

A conjunction analysis was also conducted to identify brain regions activated by both spatial and object attention in each visual quadrant. To be included in the conjunction map a given voxel had to have a significant value in the group $t$ test for spatial attention (attended vs. unattended) and object attention (same vs. different).

\section{Retinotopic Mapping}

The boundaries of retinotopically organized visual areas were determined using the method of Sereno et al. (1995). Briefly, BOLD-weighted images were obtained while subjects viewed either a slowly rotating checkerboard wedge or a dilating/expanding checkerboard ring. From these paired scans, visual field sign maps were generated in order to delineate the borders of retinotopically organized visual areas based on whether they contained a mirror-image or non-mirror-image representation of the visual field. All phase-encoded analyses of these fMRI data were carried out using the software package FreeSurfer (Dale et al., 2000; Fischl, Sereno, Dale, 1999; Fischl, Sereno, Tootell, et al., 1999; Dale \& Sereno, 1993). Initial postprocessing of fMRI data including alignment with the session structural MRI data set and motion correction was carried out with the AFNI package (Cox, 1996). Data from the attention scans described above was projected onto the rendered cortical surface containing each individuals' retinotopic boundary maps.

\section{Acknowledgments}

This work was supported by NIMH grants MH-25594 and MH67579. We thank Matt Marlow and Marina Shpaner for technical assistance.

Reprint requests should be sent to Steven A. Hillyard, Department of Neurosciences-0608, University of California, San Diego, 9500 Gilman Drive, La Jolla, CA 92093-0608, or via e-mail: shillyard@ucsd.edu.

The data reported in this experiment have been deposited in the fMRI Data Center (www.fmridc.org). The accession number is $2-2005-119 \mathrm{HH}$.

\section{REFERENCES}

Abrams, R. A., \& Law, M. B. (2000). Object-based visual attention with endogenous orienting. Perception $\mathcal{E}$ Psychophysics, 62, 818-833.

Avrahami, J. (1999). Objects of attention, objects of perception. Perception \& Psychophysics, 61, 1604-1612.

Brefczynski, J. A., \& DeYoe, E. A. (1999). A physiological correlate of the "spotlight" of visual attention. Nature Neuroscience, 2, 370-374.

Cox, R. W. (1996). AFNI-Software for analysis and visualization of functional magnetic resonance neuroimages. Computers and Biomedical Research, 29, 162-173.

Dale, A. M., Liu, A. K., Fischl, B. R., Buckner, R. L., Belliveau, J. W., Lewine, J. D., \& Halgren, E. (2000). Dynamic statistical parametric mapping: Combining fMRI and MEG for highresolution imaging of cortical activity. Neuron, 26, 55-67.

Dale, A. M., \& Sereno, M. I. (1993). Improved localization of cortical activity by combining EEG and MEG with MRI cortical surface reconstruction: A linear approach. Journal of Cognitive Neuroscience, 5, 162-176. 
Davis, G., Driver, J., Pavani, F., \& Shepherd, A. (2000). Reappraising the apparent costs of attending to two separate visual objects. Vision Research, 40, 1323-1332.

Di Russo, F., Martinez, A., \& Hillyard, S. A. (2003). Source analysis of event-related cortical activity during visuo-spatial attention. Cerebral Cortex, 13, 486-499.

Di Russo, F., Martínez, A., Sereno, M., Pitzalis, S., \& Hillyard, S. (2002). Cortical sources of the early components of the visual evoked potential. Human Brain Mapping, 15, 95-111.

Doniger, G. M., Foxe, J. J., Murray, M. M., Higgins, B. A., Snodgrass, J. G., Schroeder, C. E., \& Javitt, D. C. (2000). Activation time course of ventral visual stream objectrecognition areas: High density electrical mapping of perceptual closure processes. Journal of Cognitive Neuroscience, 12, 615-621.

Doniger, G. M., Foxe, J. J., Schroeder, C. E., Murray, M. M., Higgins, B. A., \& Javitt, D. C. (2001). Visual perceptual learning in human object recognition areas: A repetition priming study using high-density electrical mapping. Neuroimage, 13, 305-313.

Driver, J., Davis, G., Russell, C., Turatto, M., \& Freeman, E. (2001). Segmentation, attention and phenomenal visual objects. Cognition, 80, 61-95.

Egly, R., Driver, J., \& Rafal, R. D. (1994). Shifting visual attention between objects and locations: Evidence from normal and parietal lesion subjects. Journal of Experimental

Psychology: General, 123, 161-177.

Fischl, B., Sereno, M. I., \& Dale, A. M. (1999). Cortical surfacebased analysis: II. Inflation, flattening, and a surface-based coordinate system. Neuroimage, 9, 195-207.

Fischl, B., Sereno, M. I., Tootell, R. B., \& Dale, A. M. (1999). High-resolution intersubject averaging and a coordinate system for the cortical surface. Human Brain Mapping, 8, $272-284$.

Foxe, J. J., McCourt, M. E., \& Javitt, D. C. (2003). Right hemisphere control of visuospatial attention: Line-bisection judgments evaluated with high-density electrical mapping and source analysis. Neuroimage, 19, 710-726.

Foxe, J. J., Murray, M. M., \& Javitt, D. C. (2005). Filling-in in schizophrenia: A high-density electrical mapping and source-analysis investigation of illusory contour processing. Cerebral Cortex, 15, 1914-1927.

Grill-Spector, K., Kourtzi, Z., \& Kanwisher, N. (2001). The lateral occipital complex and its role in object recognition. Vision Research, 41, 1409-1422.

Grill-Spector, K., Kushnir, T., Edelman, S., Avidan, G., Itzchak, Y., \& Malach, R. (1999). Differential processing of objects under various viewing conditions in the human lateral occipital complex. Neuron, 24, 187-203.

Grill-Spector, K., Kushnir, T., Edelman, S., Itzchak, Y., \& Malach, R. (1998). Cue-invariant activation in object-related areas of the human occipital lobe. Neuron, 21, 191-202.

Grill-Spector, K., Kushnir, T., Hendler, T., Edelman, S., Itzchak, Y., \& Malach, R. (1998). A sequence of object-processing stages revealed by fMRI in the human occipital lobe. Human Brain Mapping, 6, 316-328.

He, X., Fan, S., Zhou, K., \& Chen, L. (2004). Cue validity and object-based attention. Journal of Cognitive Neuroscience, 16, 1085-1097.

Hillyard, S. A., \& Anllo-Vento, L. (1998). Event-related brain potentials in the study of visual selective attention. Proceedings of the National Academy of Sciences, U.S.A., 95, 781-787.

Hopf, J. M., Vogel, E., Woodman, G., Heinze, H. J., \& Luck, S. J. (2002). Localizing visual discrimination processes in time and space. Journal of Neurophysiology, 88, 2088-2095.

Hopfinger, J. B., Luck, S. J., \& Hillyard, S. A. (2004). Selective attention: Electrophysiological and neuromagnetic studies.
In M. S. Gazzaniga (Ed.), The cognitive neurosciences (3rd ed.). Cambridge: MIT Press.

Lamy, D., \& Egeth, H. (2002). Object-based selection: The role of attentional shifts. Perception E Psychophysics, 64 , 52-66.

Lerner, Y., Hendler, T., \& Malach, R. (2002). Object-completion effects in the human lateral occipital complex. Cerebral Cortex, 12, 163-177.

Lopez, M., Rodriguez, V., \& Valdes-Sosa, M. (2004). Two-object attentional interference depends on attentional set. International Journal of Psychophysiology, 53, 127-134.

Luck, S. J., Hillyard, S. A., Mouloua, M., Woldorff, M. G., Clark, V. P., \& Hawkins, H. L. (1994). Effects of spatial cuing on luminance detectability: Psychophysical and electrophysiological evidence for early selection. Journal of Experimental Psychology: Human Perception and Performance, 20, 887-904.

Malach, R., Reppas, J. B., Kwong, K. K., Jiang, H., Kennedy, W. A., Ledden, P. J., Brady, T. J., Rosen, B. R., \& Tootell, R. B. (1995). Object-related activity revealed by functional magnetic resonance imaging in human occipital cortex. Proceedings of the National Academy of Sciences, U.S.A., 92, 8135-8139.

Mangun, G. R. (1995). Neural mechanisms of visual selective attention. Psychophysiology, 32, 4-18.

Martinez, A., Anllo-Vento, L., Sereno, M. I., Frank, L. R., Buxton, R. B., Dubowitz, D. J., Wong, E. C., Hinrichs, H., Heinze, H. J. \& Hillyard, S. A. (1999). Involvement of striate and extrastriate visual cortical areas in spatial attention. Nature Neuroscience, 2, 364-369.

Martinez, A., Di Russo, F., Anllo-Vento, L., Sereno, M. I., Buxton, R. B., \& Hillyard, S. A. (2001). Putting spatial attention on the map: Timing and localization of stimulus selection processes in striate and extrastriate visual areas. Vision Research, 41, 1437-1457.

Maunsell, J. H., \& McAdams, C. J. (2000). Effects of attention on neuronal response properties in visual cerebral cortex. In M. S. Gazzaniga (Ed.), The new cognitive neurosciences (pp. 290-305). Cambridge: MIT Press.

McCarthy, G., \& Wood, C. C. (1985). Scalp distributions of event-related potentials: An ambiguity associated with analysis of variance models. Electroencephalography and Clinical Neurophysiology, 62, 203-208.

Moore, C. M., Yantis, S., \& Vaughan, B. (1998). Object-based visual selection: Evidence from perceptual completion. Psychological Science, 9, 104-110.

Müller, N. G., \& Kleinschmidt, A. (2003). Dynamic interaction of object- and space-based attention in retinotopic visual areas. Journal of Neuroscience, 23, 9812-9816.

Murray, M. M., Foxe, D. M., Javitt, D. C., \& Foxe, J. J. (2004). Setting boundaries: Brain dynamics of modal and amodal illusory shape completion in humans. Journal of Neuroscience, 24, 6898-6903.

Murray, M. M., Wylie, G. R., Higgins, B. A., Javitt, D. C., Schroeder, C. E., \& Foxe, J. J. (2002). The spatiotemporal dynamics of illusory contour processing: Combined highdensity electrical mapping, source analysis, and functional magnetic resonance imaging. Journal of Neuroscience, 22, 5055-5073.

Nuwer, M. R., Comi, G., Emerson, R., Fuglsang-Frederiksen, A., Guerit, J. M., Hinrichs, H., Ikeda, A., Luccas, F. J., \& Rappelsburger, T. (1998). IFCN standards for digital recording of clinical EEG. International Federation of Clinical Neurophysiology. Electroencephalography and Clinical Neurophysiology, 106, 259-261.

Pinilla, T., Cobo, A., Torres, K., \& Valdes-Sosa, M. (2001). Attentional shifts between surfaces: Effects on detection and early brain potentials. Vision Research, 41, 1619-1630. 
Ritter, W., Simson, R., \& Vaughan, H. G., Jr. (1988). Effects of the amount of stimulus information processed on negative event-related potentials. Electroencephalography and Clinical Neurophysiology, 69, 244-258.

Rose, M., Schmid, C., Winzen, A., Sommer, T., \& Buchel, C. (2004). The functional and temporal characteristics of top-down modulation in visual selection. Cerebral Cortex.

Scholl, B. J. (2001). Objects and attention: The state of the art. Cognition, 80, 1-46.

Sereno, M. I., Dale, A. M., Reppas, J. B., Kwong, K. K., Belliveau, J. W., Brady, T. J., Rosen, B. R., \& Tootell, R. B. (1995). Borders of multiple visual areas in humans revealed by functional magnetic resonance imaging. Science, 268, 889-893.

Shomstein, S., \& Yantis, S. (2002). Object-based attention: Sensory modulation or priority setting? Perception $\&$ Psychophysics, 64, 41-51.

Talairach, J., \& Tournoux, P. (1988). Co-planar stereotaxic atlas of the buman brain: 3-dimensional proportional system: An approach to cerebral imaging. New York, Thieme.

Tootell, R. B. H., Hadjikhani, N., Hall, E. K., Marrett, S., Vanduffel, W., Vaughan, J. T., \& Dale, A. M. (1998). The retinotopy of visual spatial attention. Neuron, 21, 1409-1422.

Valdes Sosa, M., Bobes, M. A., Rodriguez, V., Acosta, Y., Perez, J. I., \& Borrego, M. (2003). The influence of scene organization on attention: Psychophysics and electrophysiology. In N. Kanwisher \& J. Duncan (Eds.), Functional neuroimaging of visual cognition (pp. 321-344). Oxford, UK: Oxford University Press.

Vecera, S. P., \& Farah, M. J. (1994). Does visual attention select objects or locations? Journal of Experimental Psychology General, 123, 146-160.

Vogel, E. K., \& Luck, S. J. (2000). The visual N1 component as an index of a discrimination process. Psychophysiology, 37, 190-203.

Weber, T. A., Kramer, A. F., \& Miller, G. A. (1997). Selective processing of superimposed objects: An electrophysiological analysis of object-based attentional selection. Biological Psychology, 45, 159-182.

Wright, R. D. (1998). Visual attention (vol. 8). Oxford, UK: Oxford University Press.

Yantis, S., \& Serences, J. T. (2003). Cortical mechanisms of space-based and object-based attentional control. Current Opinion in Neurobiology, 13, 187-193. 